\title{
Cell-specific enhancement of liposomal transfection by steroids in steroid receptor expressing cells
}

\author{
FRANK KÖSTER $^{1}$, ANDREAS SCHRÖDER ${ }^{1}$, DOMINIQUE FINAS ${ }^{1}$, CHARLOTTE HAUSER $^{2}$, \\ KLAUS DIEDRICH ${ }^{1}$ and RICARDO FELBERBAUM ${ }^{1}$ \\ ${ }^{1}$ Department of Obstetrics and Gynecology, University of Lübeck, Ratzeburger Allee 160, \\ D-23538 Lübeck; ${ }^{2}$ Octagene GmbH, Am Klopferspitz 19, D-82152 Martinsried, Germany
}

Received June 19, 2006; Accepted August 30, 2006

\begin{abstract}
The DNA transfer efficiency in liposomal versus viral transfection is very low, mainly due to an insufficient nuclear transport of the delivered DNA after its endocytotic uptake to the cell. Ligand activation of intracellular steroid receptors and their subsequent mobilization to the nucleus could result in a co-transport of DNA to the nucleus. The augmentation of nuclear transport of DNA after steroid addition might cause enhanced transfection efficiency. We used cell lines from gynecologic malignoma expressing steroid receptors, such as T47D and Mcf-7 breast cancer cell lines, as well as receptor-negative cell lines, such as Hec1A from endometrium carcinoma or the breast cancer cell line MDA-MB-231. The cells were transfected by the liposomal transfection agent Dotap with the gene for firefly-luciferase as a reporter gene and transfection efficiencies were determined in the luciferase assay. We compared the effect of the addition of cholesterol and steroids in different cell lines on the transfection efficiency. The addition of cholesterol to transfection agents led to an enhancement of the luciferase activity in all cell lines. Steroids enhanced the transfection efficiency only in receptor-positive cell lines. The transfection efficiencies of HEC-1A or MDA-MB-231 cells were not enhanced by steroids. A progesterone preincubation of receptor-positive T47D cells resulted in a decrease of progesterone receptors and afterwards the progesterone enhanced transfection was dramatically diminished. We presume that the transfection enhancement by steroids is dependent on increased nuclear import of the delivered DNA only in the presence of steroid receptors. Steroid enhancement of transfection is different from the benefit of cholesterol for transfection that acts on general cellular properties or the transfection complex as such. Liposomal transfection in combination with steroids might be
\end{abstract}

Correspondence to: Dr Frank Köster, Department of Obstetrics and Gynecology, University of Lübeck, Ratzeburger Allee 160, D-23538 Lübeck, Germany

E-mail: frank-hl@web.de

Key words: breast cancer, endometrium cancer, liposomal transfection, steroids useful for a cell-specific enhancement of gene transfer for example in gynecological malignoma where subgroups are expressing high levels of steroid receptors.

\section{Introduction}

A promising strategy for gene therapy against cancer is the application of suicide genes in combination with prodrugs that must be enzymatically activated. To minimize side effects it is of great importance to target the therapeutic DNA specifically to the designated cancer cells. As a target some cases of gynecological malignoma, such as ovarian, mammarian or endometrial carcinoma, express very high levels of steroid receptors. Intracellular receptors could be involved in gene delivery. Lipoplexes composed of plasmid-DNA and cationic lipids enter the cytosolic compartment of cells via endocytosis. Only a minimum of the applied DNA reaches the nucleus, which makes nonviral transfection techniques very insufficient (1). Steroid receptors are localized in the cytoplasm and after activation by their ligand they dimerize and migrate from the cytoplasm to the nucleus where they act as transcription factors on gene promoters (2). The nuclear import of proteins is actively driven by the nuclear pore complex and is dependent on a nuclear localization signal (NLS). Such an NLS will be presented by steroid receptors after activation (3). While activated steroid receptors are migrating to the nucleus they could co-transport the delivered plasmid DNA to the nucleus by their DNA-binding capacity. Therefore, gene delivery might be improved by the addition of steroids at the point of nuclear entry.

Previous publications have reported enhanced liposomal transfection efficiency after addition of steroids (4-7). Jain et al reported that upon the addition of estradiol to lipofection the bulk of the delivered plasmid DNA was shifted from the cytoplasm into the nuclear compartment (8).

The interest in the development of nonviral transfection for gene therapy is great. While transfection efficiencies in viral gene therapy protocols are good, problems concerning immunogenic reactions and the safety of the patients make it necessary to improve nonviral techniques for gene delivery $(9,10)$. Many studies have focused on the enhancement of nuclear transport. Most protocols recommend the addition of NLS-peptides to transfection agents (11-14). Others have tried to take advantage of intracellular sources to enforce nuclear 
transport. Dean et al showed that DNA sequences with binding sites for transcription factors are preferentially shuttled into the nucleus (15). Rebuffat et al attached glucocorticoids to DNA and found an enhanced transfection specifically in cells expressing recombinant glucocorticoid receptor (16). Our approach follows the idea of a simple assay to enhance the transfection in cells expressing high levels of steroid receptors by the addition of steroids to liposomal transfection.

In this study we compared the transfection efficiency of cationic lipids alone and in combination with steroids or cholesterol on different cell lines of gynecological malignoma. The cell lines differ in their expression of the steroid receptors for progesterone (PR) and estrogen (ER). Cholesterol is customarily used in transfection protocols to stabilize the DNA liposomal complex and was previously shown to enhance Dotap transfection in cell culture in vitro and in vivo $(17,18)$. As cholesterol represents the chemical derivation of steroids it might be expected that steroids play a similar role in cellular membrane fluidity and lipoplex stabilization. By contrast, we showed previously that cholesterol and steroids have an additive effect on liposomal transfection efficiency when they are used in combination (19). Therefore we presume that there are two different mechanisms underlying the impact of steroids and cholesterol on the efficiency of transfection. While cholesterol might impair general cellular features steroids could influence transfection by the activation of their specific intracellular receptors.

\section{Materials and methods}

Cell culture. The cell lines were purchased from the European Collection of Cell Cultures (ECACC, UK). Cells were grown in cell culture flasks at $37^{\circ} \mathrm{C}$ in a $5 \% \mathrm{CO}_{2}$ humidified air atmosphere. The medium for the cell lines T47D, MCF-7, BG-1, EFO-21 and HeLa was RPMI-1640, the medium for HEC-1A and SKOV-3 was McCoy's 5A and the medium for MDA-MB-231 and BT-474 was DMEM (all media were purchased from Invitrogen, Karlsruhe). The media were completed with $10 \%$ foetal bovine serum (FCS) and antibiotics.

Transfection. One day before transfection cells were seeded on 24-well culture plates with $2 \times 10^{5}$ cells/well in full medium. Cells were washed and kept in $0.5 \mathrm{ml}$ serum containing medium without antibiotics $1 \mathrm{~h}$ before transfection. The plasmid DNA used for transfection contained a fireflyluciferase cDNA driven by a CMV-promoter and followed by an SV40 poly-A signal without SV40 enhancer sequence. Five progesterone response elements were cloned into the backbone of the plasmid. The plasmids were extracted with an Endofree Maxiprep (Qiagen, Hilden). The transfection complexes were scaled up 5-fold to be sufficient for the parallel transfection of 4 wells. DNA $(2.5 \mu \mathrm{g})$ was added to Optimem (Invitrogen, Karlsruhe) to an end volume of $50 \mu 1$. To another $50 \mu \mathrm{l}$ endvolume of Optimem, $12.5 \mu 1$ Dotap $(1 \mathrm{mg} / \mathrm{ml}$ $\mathrm{N}$-[1-(2,3-Dioleoyloxy)]-N,N,N-trimethylammonium propane methylsulfate; Carl Roth, Karlsruhe) was added. The DNAand Dotap premixes were combined carefully by pipetting and allowed to form lipoplexes for exactly $15 \mathrm{~min}$ at room temperature. Of the transfection complex, $20 \mu 1$ was applied dropwise to each well. After $20 \mathrm{~h}$ the transfection complex was exchanged against full culture medium. The luciferase activity was measured $48 \mathrm{~h}$ after the start of transfection.

Addition of steroids and cholesterol. Steroids, cholesterol and cyclodextrin were purchased as water soluble cell culture reagents from Sigma, Deisenhofen. The cyclodextrin solubilized steroids were dissolved in water in a stock solution with $26 \mathrm{mM}$ steroid concentration. Cholesterol was dissolved at a $13 \mathrm{mM}$ concentration. The cyclodextrin content varied between the steroids. To serve as a control pure cyclodextrin was prepared as a $100 \mathrm{mg} / \mathrm{ml}$ stock solution. Steroids were added to the transfection complexes together with the transfection agent before its combination with the DNA. The final concentration of steroids in the cell culture medium ranged from 5 to $150 \mu \mathrm{M}$.

Luciferase assay. Cells were lysed in $100 \mu 1$ reporter-lysis buffer (Promega, Mannheim) and frozen at $-80^{\circ} \mathrm{C}$ to perforate the membranes $48 \mathrm{~h}$ after transfection. After thawing the lysate was transferred to Eppendorf tubes and centrifuged for $1 \mathrm{~min}$ at $11000 \mathrm{x} \mathrm{g}$. Of the supernatant, $10 \mu \mathrm{l}$ was added to an opaque 96-well-plate and mixed with $50 \mu 1$ luciferase-assay (Promega, Mannheim) immediately before measurement. Analysis was performed in a Lumicount luminometer (Packard, Ontario, USA).

Correlation of transfection efficiencies. T47D cells were grown to confluence $48 \mathrm{~h}$ after transfection so we could correlate the measured luciferase activity between the wells without determination of protein contents. The results show the mean of 4 measured wells with standard deviation. On each 24-well plate we performed 4 control transfections with Dotap alone and defined the resulting value as 1.0. The transfections treated with steroids or cholesterol where correlated with the control transfections. All results represent the mean of several trials with similar results.

Western blotting. Cell lysates were produced in RIPA-buffer (1X PBS, $1 \%$ Nonidet p-40, $0.5 \%$ sodium deoxycholate, $0.1 \%$ SDS, $0.1 \mathrm{mg} / \mathrm{ml}$ PMSF, $30 \mu \mathrm{l} / \mathrm{ml}$ Aprotinin, $1 \mathrm{mM}$ sodium orthovanadate) and the supernatant was taken after $10 \mathrm{~min}$ centrifugation at $13000 \mathrm{rpm}$ at $4^{\circ} \mathrm{C}$. The protein content was determined with Bradford reagent (BioRad, Munich). Of the lysates, $15 \mu \mathrm{g}$ was analyzed in 7.5\% SDS-polyacryamide gel electrophoresis. The proteins were blotted onto a PVDF membrane (Millipore, Schwalbach) and immunodetection was performed with a mouse antibody against progesterone receptor AB25 (Santa Cruz Biotechnology, Inc., Heidelberg) and a secondary antibody coupled to horseradish peroxidase. The peroxidase activity was detected with the ECL-system (Amersham Biosciences, Freiburg).

\section{Results}

The comparison of receptor-positive and receptor-negative cells after the addition of steroids and cholesterol to Dotap transfection. The addition of cholesterol to Dotap transfection enhanced the transfection efficiency of all tested cell lines (Fig. 1). The receptor-positive T47D human breast cancer cells were enhanced 2.8 -fold and the receptor-negative cells 

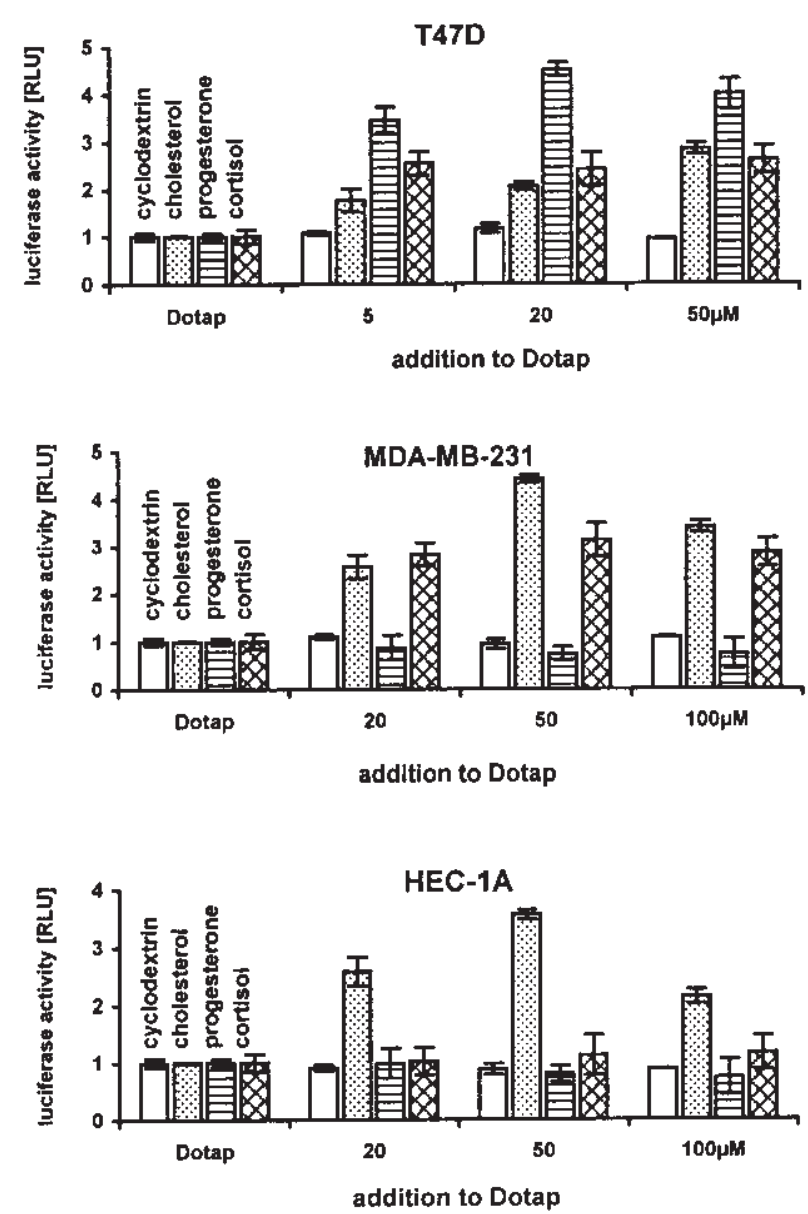

Figure 1. Cell-specific enhanced Dotap transfection with steroids. While cholesterol (dotted bars) enhanced Dotap transfection in all three cell lines, progesterone (striped bars) was only active in the receptor-positive T-47D breast cancer cells. Cortisol (diamond patterned bars) enhanced the transfection of T47D and the receptor-negative breast cancer cells MDA-MB-231. The transfection of the receptor-negative Hec1A endometrial carcinoma cell line could not be enhanced by any steroid. The luciferase activity resulting from Dotap transfection with no addition was declared as 1-fold. Cyclodextrine (open bars) was the solvent for the steroids and was used as a control, cholesterol, progesterone and cortisol were added as indicated.
HEC-1A from endometrium carcinoma and breast cancer cells MDA-MB-231 were enhanced 3.6- and 4.5-fold, respectively. The addition of progesterone enhanced the transfection efficiency in the receptor-positive T47D cells 4.51-fold but progesterone could not enhance the transfection efficiency in the receptor-negative cells HEC-1A or MDA-MB-231. We found a positive effect on transfection by the addition of cortisol in T47D cells (2.6-fold) and in MDA-MB-231 cells (4.3-fold) but only a very small effect in HEC-1A cells (1.4-fold).

Additional results are presented in Table I. T47D and MCF-7 receptor-positive breast cancer cells could also be enhanced in transfection by the steroids estrogen, cortisol and testosterone with at least 2 -fold augmentation with the exception of testosterone on MCF-7. A similar result was seen when we transfected other cell lines from gynecological carcinoma, known for positive steroid receptor expression. The breast cancer cell lines BG-1 and BT-474, the ovarian cancer cell lines SKOV-3 and EFO-21 and the cervical cancer cell line HeLa all reacted with enhanced transfection efficiency upon the addition of progesterone. Addition of estrogen, cortisol and testosterone resulted in most cases in transfection enhancement. Results are not shown, when they were not reproducible. The receptor-negative cell lines HEC-1A and MDA-MB-231 were not enhanced in transfection efficiency after addition of steroids, neither by progesterone nor by estrogen and testosterone. As an exception, cortisol augmented the transfection efficiency in MDA-MB-231 cells 3.3 -fold.

Steroid enhanced transfection after degradation of the progesterone receptor $(P R)$. The application of progesterone to the cell culture of T47D cells leads to the degradation of PR. When we incubated T47D cells with progesterone $24 \mathrm{~h}$ before transfection we detected a strongly decreased PR content in the Western blot shown in Fig. 2a. A preincubation with cholesterol did not alter the PR content. The following Dotap transfection (Fig. 2b) under the addition of progesterone or

Table I. The ER/PR receptor-positive cell lines from gynecological malignoma T47D, MCF-7, EFO-21, SKOV-3, BG-1, HeLa and BT-474 and the receptor-negative cell lines HEC-1A and MDA-MB-231 were transfected with Dotap. ${ }^{\mathrm{a}}$

\begin{tabular}{|c|c|c|c|c|c|}
\hline Cell line & Cholesterol & Progesterone & Estradiol & Cortisol & Testosterone \\
\hline \multicolumn{6}{|c|}{ ER/PR receptor-positive } \\
\hline $\mathrm{T} 47 \mathrm{D}$ & 3.5 & 4.0 & 3.0 & 2.6 & 2.9 \\
\hline MCF-7 & 3.9 & 3.7 & 4.1 & 2.0 & 1.5 \\
\hline SKOV-3 & 2.8 & 2.9 & 1.8 & 2.6 & 2.3 \\
\hline BG-1 & 4.2 & 3.4 & & 3.9 & \\
\hline EFO-21 & 3.6 & 3.0 & 1.7 & 5.3 & \\
\hline HeLa & 3.3 & 3.1 & & & 1.8 \\
\hline BT-474 & 3.0 & 2.3 & & & \\
\hline \multicolumn{6}{|l|}{ negative } \\
\hline HEC-1A & 3.6 & 1.2 & 1.1 & 1.4 & 1.1 \\
\hline MDA-MB-231 & 4.4 & 0.87 & 1.3 & 3.3 & 1.2 \\
\hline
\end{tabular}

${ }^{a}$ The results were compared with the efficiency of Dotap transfection under the addition of cholesterol and steroids. The factors by which transfection efficiency was enhanced are given. Enhancement factors $>2$ are shown in bold numbers. 


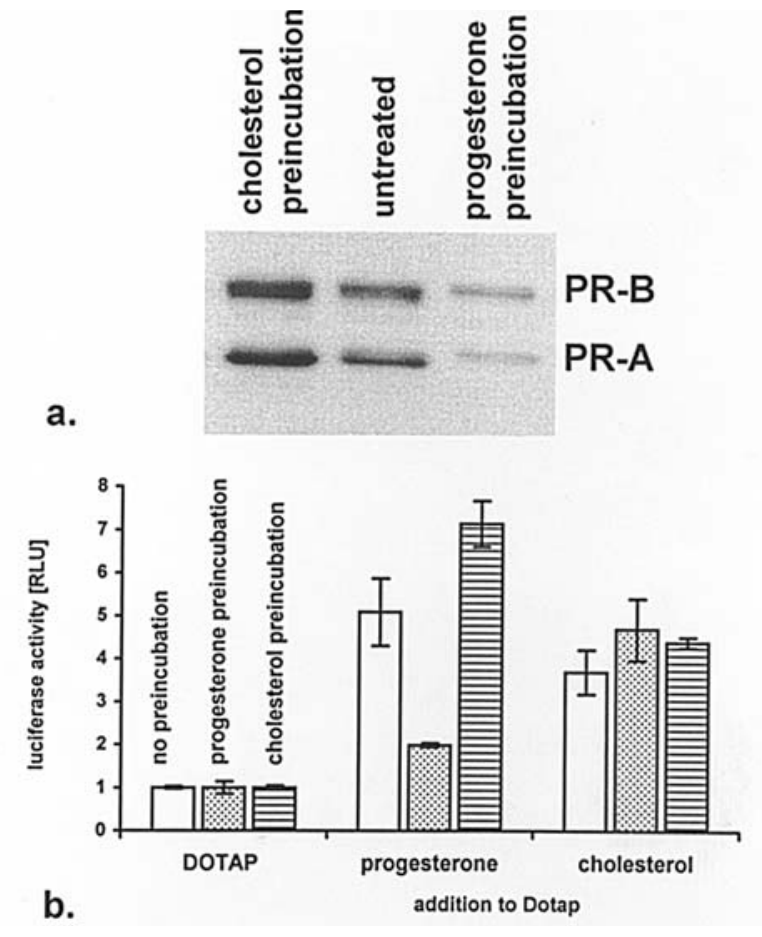

Figure 2. Progesterone enhanced transfection is reduced after degradation of the PR. (a) Progesterone preincubation for $24 \mathrm{~h}$ led to the degradation and transcriptional downregulation of the progesterone receptor in T47D cells. The Western blotting was loaded with equal amounts of protein lysates from T47D cells. Immunodetection shows a strongly decreased PR level after progesterone preincubation, but not after cholesterol preincubation (b). Without preincubation (open bars) progesterone enhanced the liposomal transfection efficiency 5.1-fold and cholesterol enhanced it 3.7-fold as shown previously. After 24-h incubation with $20 \mu \mathrm{M}$ progesterone (dotted bars) the enhancement of transfection by $50 \mu \mathrm{M}$ progesterone mixed to the transfection complex was $<2$-fold. Preincubation with $50 \mu \mathrm{M}$ cholesterol had no negative impact on the progesterone enhanced transfection (7.1-fold; striped bars).

cholesterol showed that the progesterone enhanced transfection was lowered after progesterone preincubation to 2-fold compared to 5.1-fold without preincubation but was enhanced to 7.1 -fold after cholesterol preincubation. The enhancement of Dotap transfection with the addition of cholesterol (3.7-fold) was neither negatively influenced by progesterone preincubation (4.7-fold) nor by cholesterol preincubation (4.4-fold).

\section{Discussion}

The addition of steroids to liposomal transfection enables us to enhance the transfection efficiency specifically in receptorpositive cells from gynecological malignoma. Cholesterol seems to have a ubiquitous beneficial impact on liposomal transfection. In contrast, steroids have a cell-specific influence on transfection and this is likely to be dependent on the steroid receptor status of the cell type. As HEC-1A endometrium carcinoma cells and MDA-MB-231 breast cancer cells do not express estrogen and progesterone receptors their transfection efficiency could not be increased by steroids instead of cholesterol. As an exception the glucocorticoid cortisol acts positively on liposomal transfection in MDA-MB-231 but not in HEC-1A. All tested receptor-positive cell lines were enhanced by the addition of progesterone to liposomal transfection. Furthermore, most of the receptor-positive cells were enhanced in their transfection efficiency when transfected under the addition of estrogen, cholesterol and testosterone. We conclude from these results that the steroid receptor content of the cell type is a prerequisite for the steroid enhanced liposomal transfection.

In contrast to our results, Jain et al found a positive impact of estradiol in the MDA-MB-231 cell line and concluded that the steroid receptors are not involved (8). They used a different transfection system with lipofectamine and dissolved the steroids in a polyethylene glycol and ethanol mixture, which might have functioned in a different manner. An interesting result from the study by Jain et al was that upon the addition of estradiol to liposomal transfection the plasmid DNA taken up by the cells was shifted to the nucleus (8). The nuclear import of DNA is an obstacle in liposomal transfection and it is important to ascertain whether steroids in cooperation with their receptors can be used to overwhelm this barrier.

Vacik et al reported that DNA import into the nucleus is sequence specific and dependent upon the ability of the DNA to bind to transcription factors that migrate into the nucleus (20). This makes it likely that the ligand induced movement of steroid receptors to the nucleus is responsible for the increase of the nuclear import of foreign DNA. In contrast to the data of Vacik et al it was not necessary in our tests to use a specific hormone response element (HRE) in the vector DNA sequence. We did not find a difference in the enhancement of transfection efficiency when using plasmid-DNA containing progesterone response elements (PRE) or not (21). Under natural conditions, hormone receptors recognize their HRE in the chromosomal environment specifically in cooperation with other proteins of the transcription complex (22). So it seems unlikely that the PRE in the plasmid DNA will be recognized and specifically bound by the progesterone receptor in the cytoplasm. On the other hand, steroid receptors also have an unspecific DNA binding capacity that could more likely be involved in the steroid enhanced nuclear import.

We further investigated the progesterone enhanced transfection after experimental downregulation of the progesterone receptor. After incubation of progesterone receptor-positive cells with progesterone the PR is degraded through ubiquination and S26-proteasome and additionally down-regulated on the transcriptional level (23). We found a strongly diminished transfection enhancement by progesterone addition after degradation of the PR. By contrast, the progesterone enhanced transfection was not impaired by cholesterol preincubation. Furthermore, normal transfection enhancement by cholesterol was achieved after PR downregulation. Especially the controls with cholesterol show that the preincubation process does not change the susceptibility for transfection in general and makes it feasible that the progesterone receptor content is a critical point in progesterone enhanced transfection.

Collectively, these results suggest the possibility of using steroids in a strategy for gene targeting to steroid receptor expressing cells. In the literature we found a few examples of the use of steroids to enhance liposomal transfection. Rebuffat et al coupled dexamethasone to DNA, firstly by using a spacer for covalent binding (24) and secondly by forming triple DNA helices with a PNA clamp to dexamethasone (16). In both cases they found enhanced transfection efficiency and nuclear 
transport of the DNA specifically in glucocorticoid receptor expressing cells. Recently Gruneich et al reported modified cationic dexamethasone as a transfection agent that results in high transfection efficiency mixed with DNA and the neutral lipid DOPE (dioleoylphosphatidylethanolamine) (25).

If an in vivo application of steroid enhanced transfection could be established it would be of great importance for the treatment of gynecological malignoma expressing high levels of steroid receptors.

\section{References}

1. Ma H and Diamond SL: Nonviral gene therapy and its delivery systems. Curr Pharm Biotechnol 2: 1-17, 2001

2. Baumann CT, Lim CS and Hager GL: Intracellular localization and trafficking of steroid receptors. Cell Biochem Biophys 31: 119-127, 1999.

3. Gorlich D: Transport into and out of the cell nucleus. EMBO J 17: 2721-2727, 1998.

4. Bernasconi AG, Rebuffat AG, Lovati E, Frey BM, Frey FJ and Galli I: Cortisol increases transfection efficiency of cells. FEBS Lett 419: 103-106, 1997.

5. Köster F, Felberbaum R, Finas D, Wunsch K, Schulz C, Diedrich $\mathrm{K}$ and Hauser C: Progesterone and estradiol enhance lipid mediated transfection of Sk-Br-3 mammalian cancer cells. Int J Mol Med 9: 617-620, 2002.

6. Jain PT and Gewirtz DA: Estradiol enhances gene delivery to human breast tumor cells. J Mol Med 76: 709-714, 1998.

7. Wiseman JW, Goddard CA and Colledge WH: Steroid hormone enhancement of gene delivery to a human airway epithelial cell line in vitro and mouse airways in vivo. Gene Ther 8: 1562-1571, 2001.

8. Jain PT, Seth P and Gewirtz DA: Estradiol enhances liposomemediated uptake, preferential nuclear accumulation and functional expression of exogenous genes in MDA-MB231 breast tumor cells. Biochim Biophys Acta 1451: 224-232, 1999.

9. Robertson MW III, Barnes MN, Rancourt C, Wang M, Grim J, Alvarez RD, Siegal GP and Curiel DT: Gene therapy for ovarian carcinoma. Semin Oncol 25: 397-406, 1998.

10. Hacein-Bey-Abina S, von Kalle C, Schmidt M, Le Deist F, Wulffraat N, McIntyre E, Radford I, Villeval JL, Fraser CC, Cavazzana-Calvo M, et al: A serious adverse event after successful gene therapy for X-linked severe combined immunodeficiency. N Engl J Med 348: 255-256, 2003.

11. Chan CK and Jans DA: Using nuclear targeting signals to enhance non-viral gene transfer. Immunol Cell Biol 80: 119-130, 2002.
12. Ritter W, Plank C, Lausier J, Rudolph C, Zink D, Reinhardt D and Rosenecker J: A novel transfecting peptide comprising a tetrameric nuclear localization sequence. J Mol Med 81: 708-717, 2003.

13. Subramanian A, Ranganathan P and Diamond SL: Nuclear targeting peptide scaffolds for lipofection of nondividing mammalian cells. Nat Biotechnol 17: 873-877, 1999.

14. Zanta MA, Belguise-Valladier P and Behr JP: Gene delivery: a single nuclear localization signal peptide is sufficient to carry DNA to the cell nucleus. Proc Natl Acad Sci USA 96: 91-96, 1999.

15. Dean DA, Dean BS, Muller S and Smith LC: Sequence requirements for plasmid nuclear import. Exp Cell Res 253: 713-722, 1999.

16. Rebuffat AG, Nawrocki AR, Nielsen PE, Bernasconi AG, Bernal-Mendez E, Frey BM and Frey FJ: Gene delivery by a steroid-peptide nucleic acid conjugate. FASEB J 16: 1426-1428, 2002.

17. Templeton NS, Lasic DD, Frederik PM, Strey HH, Roberts DD and Pavlakis GN: Improved DNA: liposome complexes for increased systemic delivery and gene expression. Nat Biotechnol 15: 647-652, 1997.

18. Lawrencia C, Mahendran R and Esuvaranathan K: Transfection of urothelial cells using methyl-beta-cyclodextrin solubilized cholesterol and Dotap. Gene Ther 8: 760-768, 2001.

19. Köster F, Finas D, Schulz C, Hauser C, Diedrich K and Felberbaum R: Additive effect of steroids and cholesterol on the liposomal transfection of the breast cancer cell line T-47D. Int J Mol Med 14: 769-772, 2004.

20. Vacik J, Dean BS, Zimmer WE and Dean DA: Cell-specific nuclear import of plasmid DNA. Gene Ther 6: 1006-1014, 1999.

21. Köster F, Finas D, Saupp A, Schulz C, Diedrich K, Hauser C and Felberbaum R: Enhanced liposomal transfection through the application of sex steroids in gynecological cancer cells. Zentralbl Gynakol 125: 1-5, 2003.

22. Edwards DP, DeMarzo AM, Onate SA, Beck CA, Estes PA and Nordeen SK: Mechanisms controlling steroid receptor binding to specific DNA sequences. Steroids 56: 271-278, 1991.

23. Lange CA, Shen T and Horwitz KB: Phosphorylation of human progesterone receptors at serine-294 by mitogen-activated protein kinase signals their degradation by the $26 \mathrm{~S}$ proteasome. Proc Natl Acad Sci USA 97: 1032-1037, 2000.

24. Rebuffat A, Bernasconi A, Ceppi M, Wehrli H, Verca SB, Ibrahim M, Frey BM, Frey FJ and Rusconi S: Selective enhancement of gene transfer by steroid-mediated gene delivery. Nat Biotechnol 19: 1155-1161, 2001.

25. Gruneich JA, Price A, Zhu J and Diamond SL: Cationic corticosteroid for nonviral gene delivery. Gene Ther 11: 668-674, 2004. 